\title{
The effect of Neutral Density Filters on drones orthomosaics classifications for land-use mapping
}

\author{
Jean A. Doumit ${ }^{1}$, Charbel Abou Chacra ${ }^{2}$
}

\begin{abstract}
Landscape and Street Photographers use Neutral Density (ND) Filters to enhance their photos, drones images with advanced photogrammetry software produce high-resolution orthomosaic for the production of land use maps. This paper study the effect of four different neutral density filters (ND-4, ND-8, ND-16, and ND-32) on drone orthomosaics production of a half urbanized area, a five generated orthomosaics one not filtered and four filtered were classified in a remote sensing software and compared between each other.

Three comparison methods used for the comparison between orthomosaics: An image visual interpretation, kappa index calculation for land-use quality assessment and quantity analysis of land use filter generated polygons.
\end{abstract}

Keywords: Drones, Orthomosaic, Neutral Density filters.

1. Introduction

Many drones studies were conducted for land use cartography and the fast progress in photogrammetry software encourages acquisition and processing of orthomosaics at high spatial resolutions.

The most affordable drones to be used in photogrammetry for educational and commercial purposes are the professional low prices drones.

These drones have their built-in cameras with resolutions varying from 12 to 20 megapixels depending on the drone type.

Drone images are affected by the sun rays, photogrametrists advice to make missions when the sun rays are not very high during the day, to reduce the quantity of sunlight professional photographers use Neutral Density (ND) filters.

The lack of literature and projects applying ND filters in photogrammetry gives us the opportunities to be among the first who tested the effect of these filters on orthoimages generations and their influence on the classification of land use maps.

2. Materials and methods

\footnotetext{
${ }^{1}$ Lebanese University, Faculty of Letters and Human Sciences, Department of geography, geospatial lab. jeandoumit@ul.edu.lb

${ }^{2}$ University of Saint Joseph, Beirut.
} 
The small study area of 0.25 square kilometers is a half urbanized region in Lebanon, Zouk Mikael area in Aintoura village containing diverse land use beginning from impervious surfaces such as buildings , road, trees, and herbaceous surfaces.

Neutral Density Filters, or ND filters, are referred to as neutral because they don't affect the color of light passing through the filter. photographers use ND filters to reduce the light to the sensor the same as sunglasses reduce the light to your eyes. Using a neutral density filter can decrease the amount of light by 1, 2, 3 or more stops allowing for slow shutter speed (Hanke 1979, Bryan 2004).

Table 1: neutral filters specifications (optical density, stop reduction and transmittance)

\begin{tabular}{|c|c|c|c|}
\hline $\begin{array}{c}\text { Filter } \\
\text { number }\end{array}$ & $\begin{array}{c}\text { Filter Optical } \\
\text { Density }\end{array}$ & $\begin{array}{c}\text { F/Stop } \\
\text { Reduction }\end{array}$ & $\begin{array}{c}\% \\
\text { Transmittance }\end{array}$ \\
\hline ND4 & 0.6 & 2 & $25 \%$ \\
\hline ND8 & 0.9 & 3 & $12.50 \%$ \\
\hline ND16 & 1.2 & 4 & $6.25 \%$ \\
\hline ND32 & 1.5 & 5 & $3.13 \%$ \\
\hline
\end{tabular}

Table 1 of neutral filters specification shows that the filter optical density and the stop reduction increase with filters number and have a high influence on transmittance.

An autopilot DJI Phantom 4 pro with a camera of 20 megapixels' flies between 12 and 13 o'clock when the sunshine is very high, five times the study area at the same heights of $200 \mathrm{~m}$ above the take-off point. The flight paths of all missions were identical and designed in a mobile application called Litchi, the study area and the flight parameters (coordinates, height, time, etc...). Before starting the aerial surveying, well-distinguishable 10 control points were evenly distributed within the area of interest for scaling and georeferencing the resulted data. Ground control points (GCP) were collected with the Global Positioning System (GPS) in a stereographic coordinate system.

60 aerial frames were taken from each flight with $80 \%$ overlapping and $70 \%$ side lapping, data processing repeated 5 times, the first flight with no filter added and the last four missions with filters ND-4, ND-8, ND-16 and the last mission with ND-32 filter. Captured aerial images of different filters were processed in Pix4D software for the generation of dense point clouds, Digital Surface Models (DSM) and orthomosaics 
As an output, we got five similar orthomosaic of the study area with the same spatial resolution of $7.15 \mathrm{~cm}$ (pixel size).

The generated five orthomosaics were classified with the same parameters and specifications using the K-mean clustering algorithm of the unsupervised classification method into four classes as buildings, roads, bare lands, and green areas.

Then the rasters classified land use converted to vector for quantitative analysis and comparison.

\section{Results and discussions}

Photogrammetric processing gives an output of five orthomosaics with the same spatial resolution but with different textures. the NF-no filter orthophoto is acting as a base orthomosaic for comparison between filtered orthomosaics. In a visual image interpretation of figure 1 we detected high texture details in orthomosaics ND-4, ND-8 and ND-16 otherwise in NF and ND-32 the texture is hue.

Brick roofs, tree leaves, fences, and other details are highlighted in ND-8 and ND16 and hidden in all other orthomosaics.

The variations in color brightness enable the identification of the target with other elements like shape, size, etc.

The texture is the arrangement and frequency of tonal variations from rough to smooth, smooth texture have uniform tonal variations like NF and ND-32 of figure 1. While rough texture with abruptly tonal variation in the case of ND-4, ND-8, and ND-16.
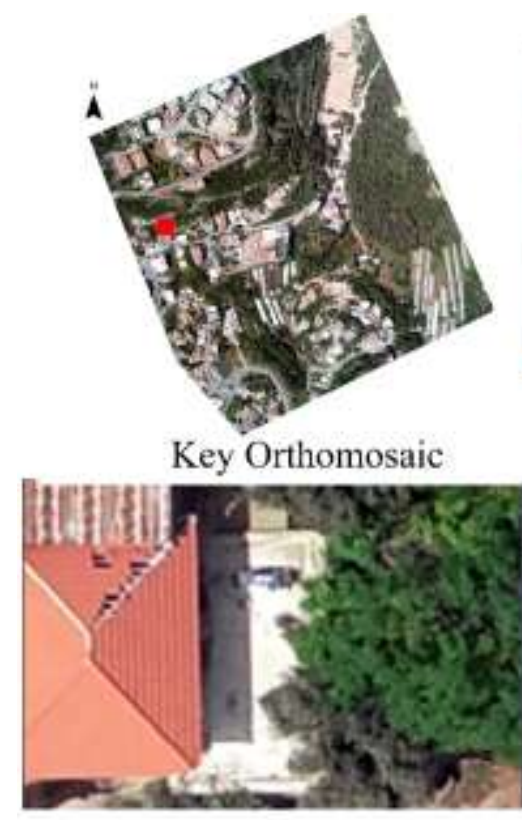

ND-8

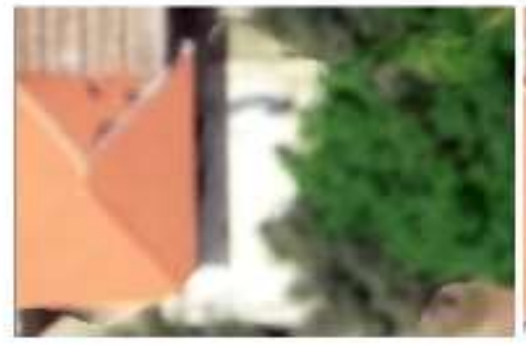

NF-without filter

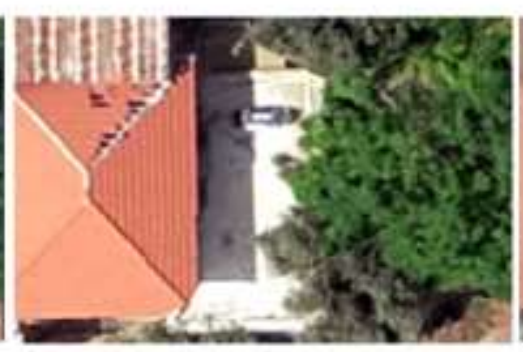

ND-16

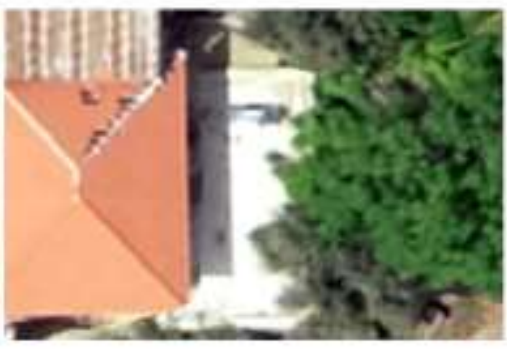

ND-4

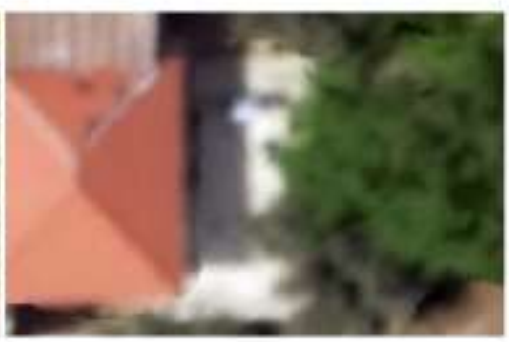

ND-32 
Fig.1. Orthomosaics generated without and with Neutral Density filters.

As a result of visual image interpretation, ND-8 and ND-16 were selected as better texture highlighting filters.

The histogram of the RGB pixel intensity provides a quantitative measure to assess the luminosity of the image. the histogram should be equally stretched across the dark and light tones (x-axes) without a peak close to 0 (underexposed) or close to 256 (overexposed) the case of all orthomosaics histograms. The graph of ND-8 is stretched along with the histogram with a middle convex shape in Red, Green and blue when the light is warm since those pixels challenging environments include brick roofs and trees leaves are highly reflective. Small textures details become visible in the small histogram peaks between luminosity values 200-256 of the image in the case of ND-4 and ND-8 of figure 2.
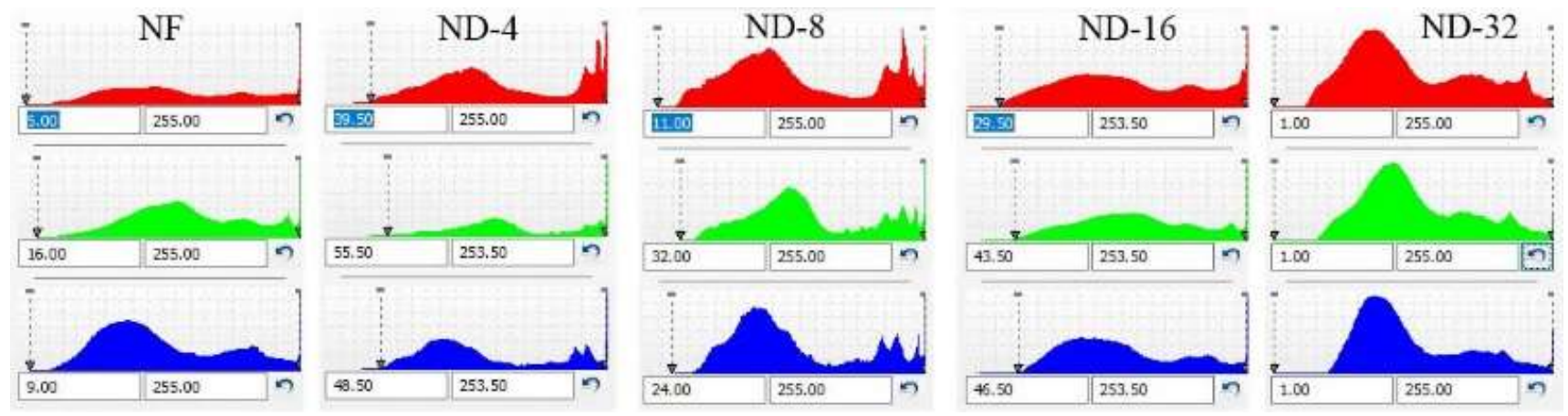

Fig.2: RGB histogram of pixels intensity of the orthomosaics.

The filter effect on land use mapping was tested by running the k-mean unsupervised classification on the five orthomosaics, table 2 of the vectorization results shows the polygons number in each land-use type.

For the verification of land use classification results of the neutral density othomosaics with 50 ground truth points measured and checked on the field. The comparison of the classification results of all Neutral density orthomosaics runs to ground truth data results in a confusion matrix and derived quality measures, more precisely overall accuracy, kappa index (Rutzinger et al., 2009), also referred to as user's and producer's accuracy.

Furthermore, the evaluation of the land use classification comprises an analysis of the influence of certain features on the classification accuracy. The confusion matrix of the result obtained by the land use classification is presented in a kappa index of 0.83 in ND-8, ND-16, and ND-4 with the same value of 0.66 and a kappa value of 0.45 .

The variation in values of the kappa index prove the visualization interpretation results, for more confirmation to this result. A quantity analysis comes to check the 
land use classification at each of the neutral density orthomosaic by converting raster land use to vector and counting land use polygons.

The land-use classification of the ND-8 filter othomosaic has a higher number of polygons in all land-use classes following by the ND-16 othomosaic then ND-4. The ND-32 orthomosaic has a lower polygons number due to the very low percentage of light transmittance which doesn't allow it to be used in photogrammetry and orthomosaic production.

Table 2: polygons numbers in each land-use type.

\begin{tabular}{|c|c|c|c|c|c|}
\cline { 2 - 6 } \multicolumn{1}{c|}{} & \multicolumn{5}{c|}{ Polygons number } \\
\cline { 2 - 6 } \multicolumn{1}{c|}{} & No-filter & ND-4 & ND-8 & ND-16 & ND-32 \\
\hline Building & 12917 & 29678 & 36222 & 32782 & 7571 \\
\hline Road & 13597 & 31006 & 38770 & 40192 & 6112 \\
\hline Bare Land & 12401 & 33144 & 42693 & 37818 & 6956 \\
\hline Green area & 4745 & 10061 & 12204 & 11796 & 2371 \\
\hline
\end{tabular}

The result of the classification step could summarize that only ND-4, ND-8, and ND-16 could be used on drones cameras for good aerial imagery.

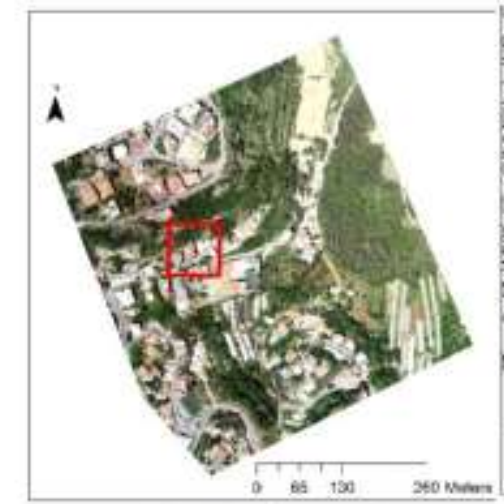

Key Orthomosaic

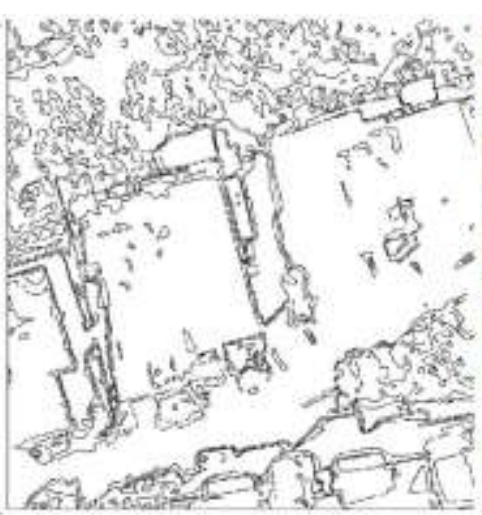

NF-without filter

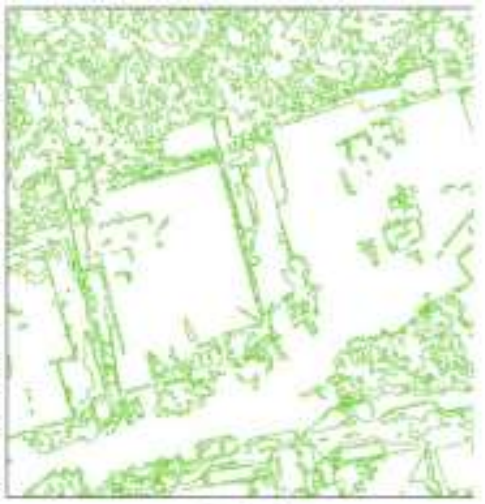

ND-4

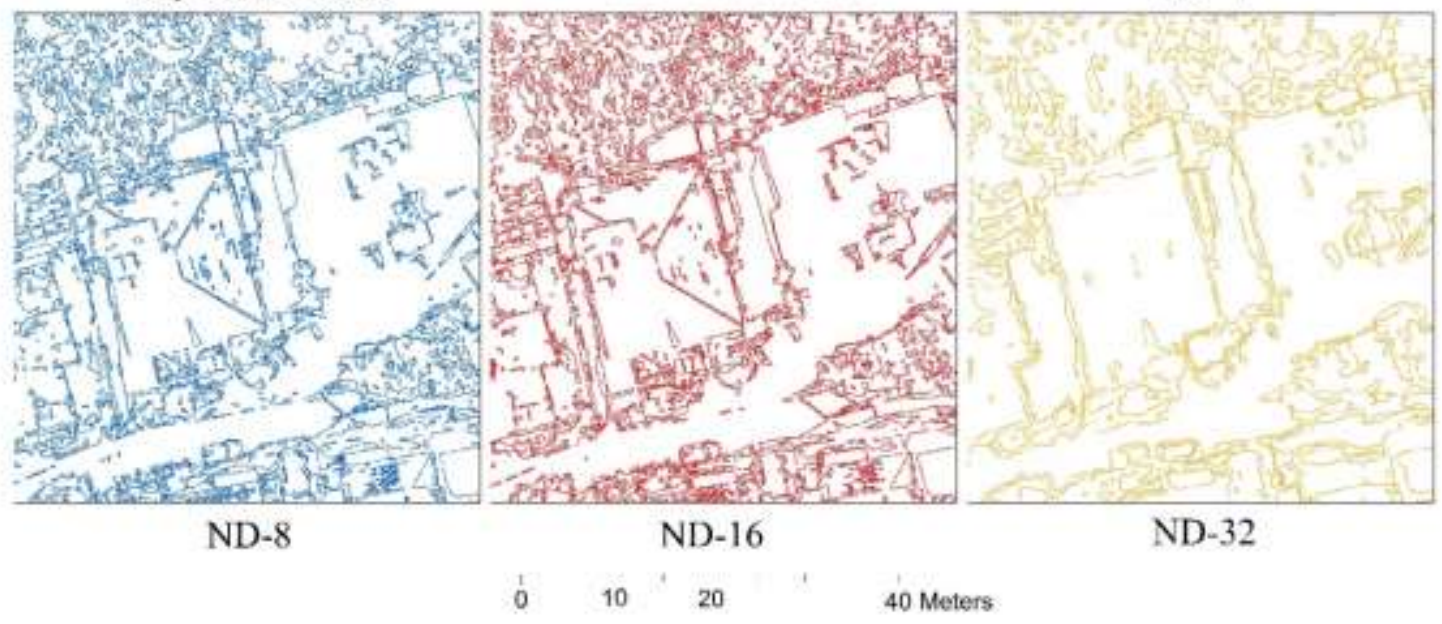

Fig.3: segmentation maps of neutral density orthomosaics 
To more understand the difference in polygons numbers between neutral filters land use maps a segmentation analysis was done, in figure 3 the skeleton map of ND-8 shows precisely the pieces of brick roof and highlight in detail linear objects such as fences and boundaries.

If we want to classify the result in image 3 by more detailed to less detailed the classification will be as follow: ND-8, ND-16, ND-4, and ND-32.

ND-32 can only be used in case of high sun rays photogrammetry missions.

\section{Conclusion}

In this paper, we studied the effect of neutral density filters for the production of orthomosaic for land-use mapping. The orthomosaics got from ND filters were visually analyzed using remote sensing image interpretation methods and using histograms.

The land use maps checked by applying the kappa index and by a quantitative analysis of the vector entity.

The general result of these analyses concludes that the use of an ND-8 filter on Dji drones gives a better quality orthomosaic for land-use mapping.

For future work, we will test ND filters on a full spectrum sensor to study the change in spectral signatures.

\section{References}

1. Bryan F.P. 2004. Understanding exposure: how to shoot great photographs with a film or digital camera.Amphoto Books. ISBN 978-08174-6300-7.

2. Hanke, R. 1979. Filter-fascination, Monheim/Bayren. P.70. ISBN 3-88324-991-2 (in German)

3. Rutzinger, M., Rottensteiner, F., Pfeifer, N., 2009. A comparison of evaluation techniques for building extraction from airborne laser scanning. IEEE Journal of Selected Topics in Applied Earth Observations and Remote Sensing 2(1), pp.11-20. 